\title{
Quantitative Graph Theory: A new branch of graph theory and network science
}

\author{
Matthias Dehmer ${ }^{1,2 *}$, Frank Emmert-Streib ${ }^{3,4 *}$, and Yongtang Shi ${ }^{5,6}$ \\ ${ }^{1}$ Department of Computer Science, Universität der Bundeswehr München, Germany \\ ${ }^{2}$ Department of Mechatronics and Biomedical Computer Science, UMIT, Hall in Tyrol, \\ Austria \\ ${ }^{3}$ Computational Medicine and Statistical Learning Laboratory, Department of Signal \\ Processing, Tampere University of Technology, Finland \\ ${ }^{4}$ Institute of Biosciences and Medical Technology, 33520 Tampere, Finland \\ ${ }^{5}$ Center for Combinatorics and LPMC-TJKLC, Nankai University, Tianjin 300071, China \\ ${ }^{6}$ College of Computer and Control Engineering, Nankai University, Tianjin 300071, China
}

\begin{abstract}
In this paper, we describe QUANTITATIVE GRAPH THEORY and argue it is a new graph-theoretical branch in network science, however, with significant different features compared to classical graph theory. The main goal of quantitative graph theory is the structural quantification of information contained in complex networks by employing a measurement approach based on numerical invariants and comparisons. Furthermore, the methods as well as the networks do not need to be deterministic but can be statistic. As such this complements the field of classical graph theory, which is descriptive and deterministic in nature. We provide examples of how quantitative graph theory can be used for novel applications in the context of the overarching concept network science.
\end{abstract}

\section{Introduction}

Graph theory [39] is a relatively new branch of mathematics. Examples of major activities include investigating topological aspects of graphs by, e.g., Kuratowski [46] and Gross [36], leading to the emergence of topological graph theory [36, studying embeddings of graphs in surfaces and graphs as topological spaces. A highlight in this theory is surely the theorem of Kuratowski for characterizing planar graphs. Further activities contributed numerous methods for exploring structural properties of networks, e.g., see $9,20,38,39$. Note that there are also other branches of Graph Theory, such as Extremal or Random Graph Theory, which have also been very fruitful [9].

Based on those multifaceted methods, numerous problems by means of graphs have been solved in disciplines such as artificial intelligence and pattern recognition 17], biology [33, 43, chemoinformatics [58, 59, 71, cognitive modeling 65], computational linguistics [51, image recognition [41, 66, machine learning $[12,40]$ and web mining [25].

In a wider sense, graph-based approaches have been used extensively in various disciplines. The hype dealing with complex networks also contributed a lot to modern Graph or Network Theory and has been triggered from the breakthrough of the world wide web and other physically-oriented studies exploring networks (graphs) as complex systems [4,73. Besides investigating only random graph models for analyzing complex systems, it turned out that there is a strong need to further develop quantitative approaches. A main reason for this was the insight that many real-world networks are composed of non-random topologies where quantitative methods such as graph measures 19,20 have been proven essential to quantify structural information of graphs.

When studying the existing literature dealing with classical aspects of graph theory [38, 39], it turns out that most of the existing contributions are descriptive approaches for describing graphs structurally. Examples thereof are Kuratowski's theorem mentioned above, the description of Eulerian paths, graph colorings and so forth $7,3,38,39$. This leads us straightforwardly to a definition of Quantitative Graph Theory:

Definition 1.1 Quantitative graph theory (QGT) deals with the quantification of structural aspects of graphs, instead of characterizing graphs only descriptively, 
We see that the aspect of measurement is crucial here. To quantify structural information of a graph means to employ any kind of measurement, i.e., a local or global one.

Concrete examples for quantitative approaches in the context of Graph Theory are, for instance:

- Graph similarity or distance measures $25,64,76$.

- Graph measures to characterize the topology of a graph [19,20,68. This group of measurements includes numerical graph invariants which are also referred to as topological indices 11, 31.

- The exploration of metrical properties of graphs and the derivation of measures 63].

- Consideration of structural graph measures as network complexity measures and exploring properties thereof 10,29 .

- Information Theory and Statistics of graphs 5, 5,45 .

- Applications of the above graph measures in social network analysis, chemistry, biology and other disciplines 31,72 .

- Using machine learning for deriving quantitative approaches for graphs [18.

As known, various graph measures have been defined and investigated 19,20 even in the early fifties 6 . Seminal contributions from this development have been graph centrality measures for investigating problems in sociology, e.g., group performance 6]. Numerous other graph-theoretical measurements have been developed afterwards, but to date, there have been no attempts to consider Quantitative Graph Theory as a graph-theoretical branch. In view of the vast number of existing descriptive methods $7,38,39$, quantitative techniques to analyze graphs are clearly underrepresented so far. However, quantitative approaches for exploring graphs have been examined from different perspectives in a variety of disciplines including Discrete Mathematics, Computer Science, Biology, Chemistry and related areas 21,43. Interestingly, in Biology, Computer Science and Discrete Mathematics, the focus has tended to be on the comparative analysis of graphs such as graph similarity and graph distances. In contrast, in Chemistry and related fields, the principal aim has been to quantify structural features of graphs leading to numerical graph invariants 32 .

\section{Quantitative Graph Theory}

In 24], Quantitative Graph Theory has been defined as a measurement approach to quantify structural information of networks. In general, local, global or comparative graph measures can be employed for measuring structural information. See also Section 3. We emphasize that this definition complements (classical) graph theory which mainly deals with the description of structural properties of graphs, see [39]. Examples for pure descriptive graph theory methods are graph colorings, graph embeddings and decompositions, and characterizations of graphs like the Theorem of Kuratowski [4]. In contrast, pure quantitative methods are quantitative graph measures that are based on estimating structural features of networks 20]. The latter class of methods can be interpreted as graph complexity measures. Seminal work in this area was done by Mowshowitz [52 and Bonchev [10]. Note that the ambiguity of some methods belonging to both Descriptive and Quantitative Graph Theory has been explained in [24.

In Figure 1, we present a conceptual overview that shows the connection of Quantitative Graph Theory within some other related fields. First, we note that Network Science comprises both, Graph Theory and Statistical Network Analysis because it is the most generic field. The major difference between Graph Theory and Statistical Network Analysis is that the graphs as well as the methods of Graph Theory are purely deterministic, whereas in statistical network analysis the networks and the methods may be non-deterministic. A cause for the structural non-deterministicness may be given by, e.g., measurement 


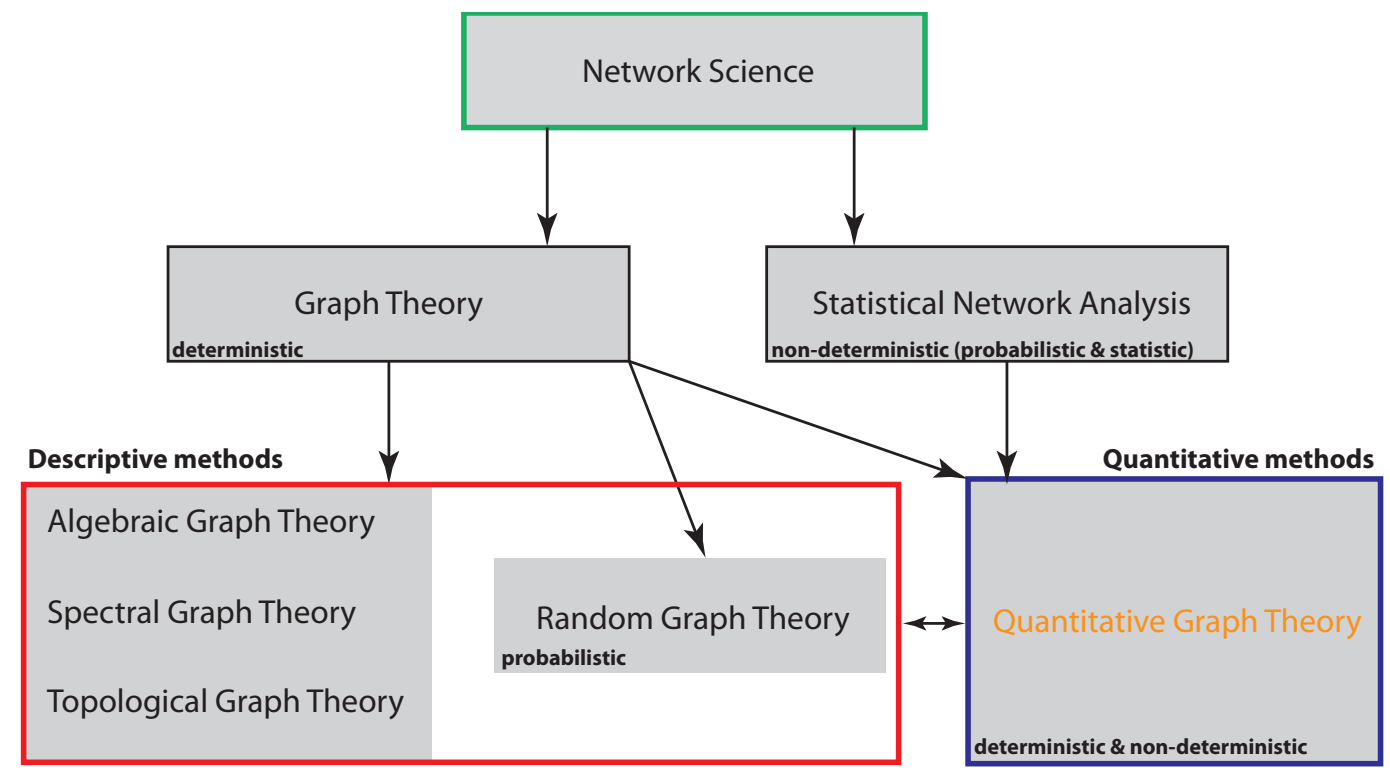

Figure 1. Quantitative graph theory as a branch of graph theory and network science.

errors or signal variability used to infer such networks 33. Hence, the methods applied are based on probabilistic and statistical principles. Furthermore, Quantitative Graph Theory is also a branch of Graph Theory which relates to quantify structural information of networks. This is in contrast to classical Graph Theory which mainly deals with descriptive graph analysis, as discussed above. Interestingly, one can see that random graph theory is on one hand deterministic, when describing properties of random graphs. On the other hand, the methods and the random graphs themselves are often probabilistic and, hence, this subfield is also non-deterministic in nature.

\section{Methods Used in Quantitative Graph Theory}

According to Dehmer et al. 24], Quantitative graph theory can be divided into two major categories, namely, comparative graph analysis 24 and graph characterization by using numerical graph invariants is another category which relates to determine the structural complexity of networks. In the following, we give some prominent examples of methods for these two categories. For more details see [24].

\subsection{Comparative Graph Analysis}

Comparative graph analysis means determining the structural similarity or distance between two or more networks. A problem has been choosing the right concept for this task meaningfully as a graph similarity measure depends on an underlying concept. When doing so, two main classes thereof namely (exact graph matching) [76] and inexact matching [14 to compare networks structurally have been developed. In particular, methods from exact graph matching have been demanding to use for large networks as the measures rely on graph isomorphism. As known, the complexity of the graph isomorphism has not yet determined for arbitraty networks.

Comparative techniques for networks have been widely applied in many scientific disciplines. Examples are artificial intelligence and pattern recognition [17, computational biology [33, 43, chemoinformatics [58,71], image recognition [41,66, and applied mathematics 22, 25]. A subset thereof is: 
- Isomorphism-based Measures $44,64,76$.

- Graph Edit Distance 13

- Iterative Graph Similarity Methods 8.75

- String-based Measures $25,34,60$

- Graph Kernels 12,40

\subsubsection{Applications}

As many tasks in machine learning rely on similarity concepts, it has been straightforward to use graph similarity (comparative network analysis) for applications. For instance, graph similarity measures can be used to compare and to classify structures representing real objects which can be visualized as relational structures. When doing so, it has been also important to use graph similarty concepts in machine learning as the underlying algorithms are often rely on similarity between objects to be processed. For instance, the following application areas turned out to be useful and important:

- Similarity of Document Structures [15,26].

- Tree Similarity 42,61

- Molecular Similarity 49,59 .

- Statistical Graph Matching 62,67.

It is obvious that for some specific problems, one needs special measures which are not require to operate on the entire network. An example for this are tree similarity measures 42,61 that operate on trees only representing connected and acyclic networks. Using such special comparative measures may be beneficial to cluster document or RNA structures that are represented by trees only. This gives us an idea about the complexity of the network similarity problem as various graph classes exist when it comes to realize a particular application.

\subsection{Graph Characterization}

In quantitative graph theory [24], graph characterization relates to determine the complexity of a given network by using numerical graph invariants. Graph invariants are graph measures to characterize graphs structurally which are invariant under isomorphism 68 . Examples for local graph invarinats are vertex degrees or vertex eccentricities [39 63]; global graph invariants are, for instance, graph entropy measures or measures which are based on distances in networks like the well-known Wiener index [68]. All those measures have in common that they are functions which map networks to the reals 68 . A special kind of graph measures are so-called topological indices 68. Note that they have been used in mathematical and structural chemistry extensively to characterize molecular structures based on their size, shape, branching and cyclicity [68. Importantly, an undesired aspect of all structural graph measures is their degeneracy 23 68]; that means, many of these graphs measures fail to discriminate the structure of nonisomorphic graphs by their values uniquely. This property often has a negative effect when determining the complexity of networks numerically. Therefore it has been important to investigate the problem on a large scale to finally obtain a classification of graph measures according to their properties such as degeneracy, structural interpretation and so forth, see 23.68. Based on graph invariants to be used, graph measures can be roughly divided into the following classes:

- Distance-based graph measures. Examples: Wiener index, eccentrcity 63.74. 
- Degree-based measures. Examples: Randić index and Zagreb index [55, 57].

- Eigenvalue-based measures. Examples: Graph energy and largest positive eigenvalue of trees [4748].

- Information-theoretic measures. Examples: Vertex-orbit entropy due to Mowshowitz and partitionindependent graph entropies $[28$.

As to applications, these graph measures have been used in chemoinformatics (e.g., QSAR and QSPR) [68 for predicting biological or physico-chemical properties of molecular structures 31, 68. Also, structural graph measures have been employed to analyze phylogenetic properties of metabolic networks [50. Moreover, graph measures got attention from areas outside chemistry and biology as structural systems representing networks occur in many other disciplines too. Examples are computational linguistics 3 and web structure mining [16] where web-based units have been explored by using structiral indices.

For applications, it turned out to be important understanding the properties of graph measures properly. Otherwise, the sage of those measure might misleading. We here summarize some important properties as follows:

- Uniqueness of graph measures (i.e., degeneracy) 23,68.

- Information inequalities describing interrelations of information-theoretic network measures 27].

- Structural interpretation 24 .

- Usefulness and quality of graph measures 24,35 .

- Correlation of graph measures 24.

\subsection{Software for Quantitative Graph Analysis}

In this section, we briefly discuss free and comercial software to analyze complex networks.

\subsubsection{Programs developed for the Statistical programming Language $R$}

The multiparadigm language $\mathrm{R} 2$ turned out to be useful for analyzing networks from various disciplines. An example is the R-package NetBioV $[70$ for visualizing large biological networks. Other standard Rlibraries for structural network analysis are igraph and graph. They contain many methods like the calculation of shortest paths in networks. However they only offer a very few quantitative methods for characterizing networks. For this purpose, the R-package QuACN [53] has been developed which contains over 150 structural network measures. Further R-package for performing network analysis can be found in 24,54 .

\subsubsection{Commercial Software}

A well-kown example of commercial software for claculating structural and molecular indices (measures) is Dragon 69]. Actually it contains more than 4000 molecular descriptors 68] for characterizing molecular networks. This software has been proven useful for QSAR and QSPR 31]. Other commercial pieces of software are, e.g., the Sentinel Visualizer [37] and Sonamine [1]. Sentinel Visualizer 37] is a software for analyzing and visualizing social networks. Sonamine 1 has been developed for performing data mining and simulation techniques for massive social networks. 


\subsubsection{Conjecture engines}

Conjecture engines [30,56] are tools that have been used to generate conjectures when proving statements on topological graph measures. They can be used to test the validity of inequalities involving topoological graph measures. The tool Graffiti 30 is a prominent example and has been famous for generating conjectures involving the general and simple Randić index. A succesor thereof is GrInvIn [56] which is a freely available as Java-Application A shortcoming of GrInvIn is the quite weak conjecture engines which does not allow to use this tool to explore complex conjectures.

\section{Summary and Conclusion}

In this chapter, we put the emphasis on quantitative graph theory. We pointed out that this intriguing field can be seen as a new branch of graph theory and should be treated as such. So far, most of the contributions in graph theory dealt with descriptive approaches for describing structural features of networks, see Section 1. By demonstrating the potential of quantitative approaches which have been widely spread over several disciplines, we believe that the discussed approaches will break new ground. An important finding of this chapter is that quantitative graph theory is per se strongly interdisciplinary. The mathematical apparatus belongs to applied mathematics but, as demonstrated, quantitative graph theory have been applied in various scientific areas.

\section{Acknowledgments}

Matthias Dehmer thanks the Austrian Science Funds for supporting this work (project P26142). Matthias Dehmer gratefully acknowledges financial support from the German Federal Ministry of Education and Research (BMBF) (project RiKoV, Grant No. 13N12304).

\section{References}

1. Sonamine. http://www.sonamine.com/home/, 2009.

2. R, software, a language and environment for statistical computing. www.r-project.org, 2011. R Development Core Team, Foundation for Statistical Computing, Vienna, Austria.

3. O. Abramov and T. Lokot. Typology by means of language networks. applying information theoretic measures to morphological derivation networks. In M. Dehmer, F. Emmert-Streib, and A. Mehler, editors, Towards an Information Theory of Complex Networks: Statistical Methods and Applications, pages 321-346. Birkhäuser, Boston/Basel, 2011.

4. R. Albert, H. Jeong, and A. L. Barabási. Diameter of the world wide web. Nature, 401:130-131, 1999.

5. K. Anand and G. Bianconi. Entropy measures for networks: Toward an information theory of complex topologies. Phys. Rev. E, 80:045102(R), 2009.

6. A. Bavelas. Communication patterns in task-oriented groups. J. Acoust. Soc. Amer., 22:725-730, 1950.

7. M. Behzad, G. Chartrand, and L. Lesniak-Foster. Graphs \& Digraphs. International Series. Prindle, Weber \& Schmidt, 1979. 
8. V. Blondel, A. Gajardo, M. Heymans, P. Senellart, and P. V. Dooren. A measure of similarity between graph vertices: applications to synonym extraction and web searching. SIAM Review, 46:647-666, 2004.

9. B. Bollabás. Modern Graph Theory. Graduate Texts in Mathematics. Springer, New York, 1998.

10. D. Bonchev. Information theoretic measures of complexity. In R. Meyers, editor, Encyclopedia of Complexity and System Science, volume 5, pages 4820-4838. Springer, 2009.

11. D. Bonchev and D. H. Rouvray. Chemical Graph Theory. Introduction and Fundamentals. Abacus Press, 1991. New York, NY, USA.

12. M. Borgwardt. Graph Kernels. PhD thesis, Ludwig-Maximilians-Universität München, Fakultät für Mathematik, Informatik und Statistik, 2007.

13. H. Bunke. What is the distance between graphs ? Bulletin of the EATCS, 20:35-39, 1983.

14. H. Bunke. Recent developments in graph matching. In 15-th International Conference on Pattern Recognition, volume 2, pages 117-124, 2000.

15. D. Buttler. A short survey of document structure similarity algorithms. In International Conference on Internet Computing, pages 3-9, 2004.

16. S. Chakrabarti. Mining the Web: Discovering Knowledge from Hypertext Data. Morgan Kaufmann, San Francisco, 2002.

17. D. Conte, F. Foggia, C. Sansone, and M. Vento. Thirty years of graph matching in pattern regocnition. International Journal of Pattern Recognition and Artificial Intelligence, 18:265-298, 2004.

18. D. Cook and L. B. Holder. Mining Graph Data. Wiley-Interscience, 2007.

19. L. da F. Costa, F. Rodrigues, and G. Travieso. Characterization of complex networks: A survey of measurements. Advances in Physics, 56:167-242, 2007.

20. M. Dehmer, editor. Structural Analysis of Complex Networks. Birkhäuser Publishing, 2010.

21. M. Dehmer and F. Emmert-Streib, editors. Analysis of Complex Networks: From Biology to Linguistics. Wiley VCH Publishing, 2009.

22. M. Dehmer, F. Emmert-Streib, and Y. Shi. Interrelations of graph distance measures based on topological indices. PLoS ONE, 9:e94985, 2014.

23. M. Dehmer, M. Grabner, and K. Varmuza. Information indices with high discriminative power for graphs. PLoS ONE, 7(2), 2012. doi: 10.1371/journal.pone.0031214.

24. M. Dehmer, V. Kraus, F. Emmert-Streib, and S. Pickl. What is Quantitative Graph Theory ?, pages 1-33. CRC Press, 2014.

25. M. Dehmer and A. Mehler. A new method of measuring similarity for a special class of directed graphs. Tatra Mountains Mathematical Publications, 36:39-59, 2007.

26. M. Dehmer, A. Mehler, and F. Emmert-Streib. Graph-theoretical characterizations of generalized trees. In Proceedings of the International Conference on Machine Learning: Models, Technologies E Applications (MLMTA'07), Las Vegas/USA, 2007, pages 113-117, 2007. 
27. M. Dehmer and A. Mowshowitz. Inequalities for entropy-based measures of network information content. Appl. Math. Comput., 215:4263-4271, 2010.

28. M. Dehmer and A. Mowshowitz. A History of Graph Entropy Measures. Inf. Sci., 1:57-78, 2011.

29. M. Dehmer, A. Mowshowitz, and F. Emmert-Streib. Connections between classical and parametric network entropies. PLoS ONE, 6:e15733, 2011.

30. E. DeLaVina. Some history of the development of graffiti. DIMACS Series in Discrete Mathematics and Theoretical Computer Science 69: Graphs and Discovery, pages 81-118, 2005.

31. J. Devillers and A. T. Balaban. Topological Indices and Related Descriptors in QSAR and QSPR. Gordon and Breach Science Publishers, 2000. Amsterdam, The Netherlands.

32. M. V. Diudea, I. Gutman, and L. Jäntschi. Molecular Topology. Nova Publishing, 2001. New York, NY, USA.

33. F. Emmert-Streib. The chronic fatigue syndrome: A comparative pathway analysis. Journal of Computational Biology, 14(7), 2007.

34. F. Emmert-Streib, M. Dehmer, and J. Kilian. Classification of large graphs by a local tree decomposition. In H. R. A. et al., editor, Proceedings of DMIN'05, International Conference on Data Mining, Las Vegas, USA, pages 200-207, 2006.

35. B. Furtula, I. Gutman, and M. Dehmer. On structure-sensitivity of degree-based topological indices. Applied Mathematics and Computation, 219:8973-8978, 2013.

36. J. L. Gross and T. W. Tucker. Topological graph theory. Wiley Interscience, 1987.

37. F. A. S. Group. Sentinel visualizer. www.fmsasg.com, 2015.

38. R. Halin. Graphentheorie. Akademie Verlag, 1989. Berlin, Germany.

39. F. Harary. Graph Theory. Addison Wesley Publishing Company, 1969. Reading, MA, USA.

40. T. Horváth, T. Gärtner, and S. Wrobel. Cyclic pattern kernels for predictive graph mining. In Proceedings of the 2004 ACM SIGKDD International Conference on Knowledge Discovery and Data Mining, pages 158-167, 2004.

41. S. M. Hsieh and C. C. Hsu. Graph-based representation for similarity retrieval of symbolic images. Data Knowl. Eng., 65(3):401-418, 2008.

42. T. Jiang, L. Wang, and K. Zhang. Alignment of trees - an alternative to tree edit. In $C P M$ '94: Proceedings of the 5th Annual Symposium on Combinatorial Pattern Matching, pages 75-86, London, UK, 1994. Springer-Verlag.

43. B. H. Junker and F. Schreiber. Analysis of Biological Networks. Wiley Series in Bioinformatics. Wiley-Interscience, 2008.

44. F. Kaden. Graphmetriken und Distanzgraphen. ZKI-Informationen, Akad. Wiss. DDR, 2(82):1-63, 1982.

45. E. D. Kolaczyk. Statistical Analysis of Network Data. Springer Series in Statistics. Springer, 2009.

46. K. Kuratowski. Sur le problème des courbes gauches en topologie. Fund. Math. Vol., 15:271-283, 1930. 
47. X. Li, Y. Shi, and I. Gutman. Graph Energy. Springer, 2012. New York.

48. L. Lovász and J. Pelikán. On the eigenvalues of trees. Periodica Mathematica Hungarica, 3(12):175-182, 1973.

49. G. M. Maggiora and V. Shanmugasundaram. Molecular similarity measures. In Chemoinformatics: Concepts, Methods, and Tools for Drug Discovery, pages 1-50. Humana Press, 2004. Totowa, NJ, USA.

50. A. Mazurie, D. Bonchev, B. Schwikowski, and G. A. Buck. Phylogenetic distances are encoded in networks of interacting pathways. Bioinformatics, 24(22):2579-2585, 2008.

51. A. Mehler, P. Weiß, and A. Lücking. A network model of interpersonal alignment. Entropy, 12(6):1440-1483, 2010.

52. A. Mowshowitz. Entropy and the complexity of the graphs I: An index of the relative complexity of a graph. Bull. Math. Biophys., 30:175-204, 1968.

53. L. Müller, M. Schutte, K. G. Kugler, and M. Dehmer. QuACN: Quantitative Analyze of Complex Networks, 2012. R Package Version 1.6.

54. L. A. J. Müller, M. Dehmer, and F. Emmert-Streib. Computational Medicine, chapter Networkbased Methods for Computational Diagnostics by Means of R, pages 185-197. Springer, 2012.

55. S. Nikolić, G. Kovačević, A. Miličević, and N. Trinajstić. The Zagreb Index 30 Years After. Croat. Chem. Acta, 76:113-124, 2003.

56. A. Peeters, K. Coolsaet, G. Brinkmann, N. Cleemput, and V. Fack. Grinvin in a nutshell. Journal of Mathematical Chemistry, 45:471-477, 2009.

57. M. Randić. On Characterization of Molecular Branching. J. Amer. Chem. Soc., 97:6609-6615, 1975.

58. M. Randić. Design of molecules with desired properties. molecular similarity approach to property optimization. In M. A. Johnson and G. Maggiora, editors, Concepts and Applications of Molecular Similarity, pages 77-145. Wiley, 1990.

59. M. Randić and C. L. Wilkins. Graph theoretical approach to recognition of structural similarity in molecules. J. Chem. Inf. Comput. Sci., 19:31-37, 1979.

60. A. Robles-Kelly and R. Hancock. Edit distance from graph spectra. In Proceedings of the IEEE International Conference on Computer Vision, pages 234-241, 2003.

61. S. M. Selkow. The tree-to-tree editing problem. Inf. Process. Lett., 6(6):184-186, 1977.

62. L. B. Shams, M. J. Brady, and S. Schaal. Graph matching vs mutual information maximization for object detection. Neural Networks, 14(3):345-354, 2001.

63. V. A. Skorobogatov and A. A. Dobrynin. Metrical Analysis of Graphs. MATCH Commun. Math. Comp. Chem., 23:105-155, 1988.

64. F. Sobik. Graphmetriken und Klassifikation strukturierter Objekte. ZKI-Informationen, Akad. Wiss. DDR, 2(82):63-122, 1982.

65. E. Sommerfeld. Systematization and formalization of cognitive structure transformations on the basis of graph transformations. In T. Marek, editor, Action and performance: Models and tests. Contributions to the quantitative psychology and its methodology, pages 105-120. 1990. 
66. C. Theoharatos, N. Laskaris, G. Economou, and S. Fotopoulos. A similarity measure for color image retrieval and indexing based on the multivariate two sample problem. In Proceedings of EUSIPCO, Vienna, Austria, 2004.

67. C. Theoharatos, V. K. Pothos, N. A. Laskaris, G. Economou, and S. Fotopoulos. Multivariate image similarity in the compressed domain using statistical graph matching. Pattern Recognition, 39(10):1892-1904, 2006.

68. R. Todeschini, V. Consonni, and R. Mannhold. Handbook of Molecular Descriptors. Wiley-VCH, 2002. Weinheim, Germany.

69. R. Todeschini, V. Consonni, A. Mauri, and M. Pavan. Dragon, software for calculation of molecular descriptors. www.talete.mi.it, 2004. Talete srl, Milano, Italy.

70. S. Tripathi, M. Dehmer, and F. Emmert-Streib. Netbiov: an r package for visualizing large network data in biology and medicine. Bioinformatics, 19:2834-2836, 2014.

71. K. Varmuza and H. Scsibrany. Substructure isomorphism matrix. J. Chem. Inf. Comput. Sci., 40:308-313, 2000.

72. S. Wasserman and K. Faust. Social Network Analysis: Methods and Applications. Structural Analysis in the Social Sciences. Cambridge University Press, 1994.

73. D. J. Watts and S. H. Strogatz. Collective dynamics of 'small-world' networks. Nature, 393:440-442, 1998.

74. H. Wiener. Structural determination of paraffin boiling points. Journal of the American Chemical Society, 69(17):17-20, 1947.

75. L. A. Zager and G. C. Verghese. Graph similarity scoring and matching. Applied Mathematics Letters, 21:86-94, 2008.

76. B. Zelinka. On a certain distance between isomorphism classes of graphs. Časopis pro $\breve{\text { pest. }}$ Mathematiky, 100:371-373, 1975. 